Supplementary Information for:

\title{
Transport and attenuation of particles of different density and surface charge: a karst aquifer field study
}

\author{
Ferry Schiperski, ${ }^{*}$ Johannes Zirlewagen, and Traugott Scheytt \\ Technische Universität Berlin, Dept. of Applied Geosciences, Hydrogeology Research \\ Group, 10587 Berlin, Germany \\ E-mail: schiperski@tu-berlin.de \\ Phone: $+49(0) 3031472652$
}




\section{Contents of this file}

Number of pages: 5

Number of Figures: 2

Number of Tables: 1

\section{List of Tables}

S1 Tap water and spring water chemistry at the test site during stable discharge conditions of $0.8 \mathrm{~m}^{3} \mathrm{~s}^{-1}$ in April 2015. . . . . . . . . . . . . . S3

\section{List of Figures}

S1 Probability density functions of particle distributions on filters for three examples with mean particle concentrations per image $(\bar{x})$ of $(\mathrm{A}) 4.2$, (B) 2.3, and (C) 0.6. Calculated values for a Kolmogorov-Smirnov test are well above the critical values at a significance level of $\alpha=5 \%$ and $n=400(\lambda=$ standard deviation; $\mathrm{a}_{o b s}=$ Kolmogorov-Smirnov values; $\mathrm{a}_{\text {crit }}=$ critical KolmogorovSmirnov values). . . . . . . . . . . . . . . . . .

S2 Observed (obs) versus nominal standard deviations (stdev) of particle enumeration for each of the analyzed filters. Filled symbols show analysis that were considered for further processing, unfilled symbols show analysis that were excluded $\left(\mathrm{Si}^{0}\right.$ : unmodified silicate particles; $\mathrm{Si}^{\mathrm{COOH}}$ : carboxylated silicate particles; $\mathrm{PS}^{0}$ : unmodified polystyrene particles; $\mathrm{PS} C O O H$ : carboxylated polystyrene particles $\ldots \ldots \ldots \ldots \ldots$ 


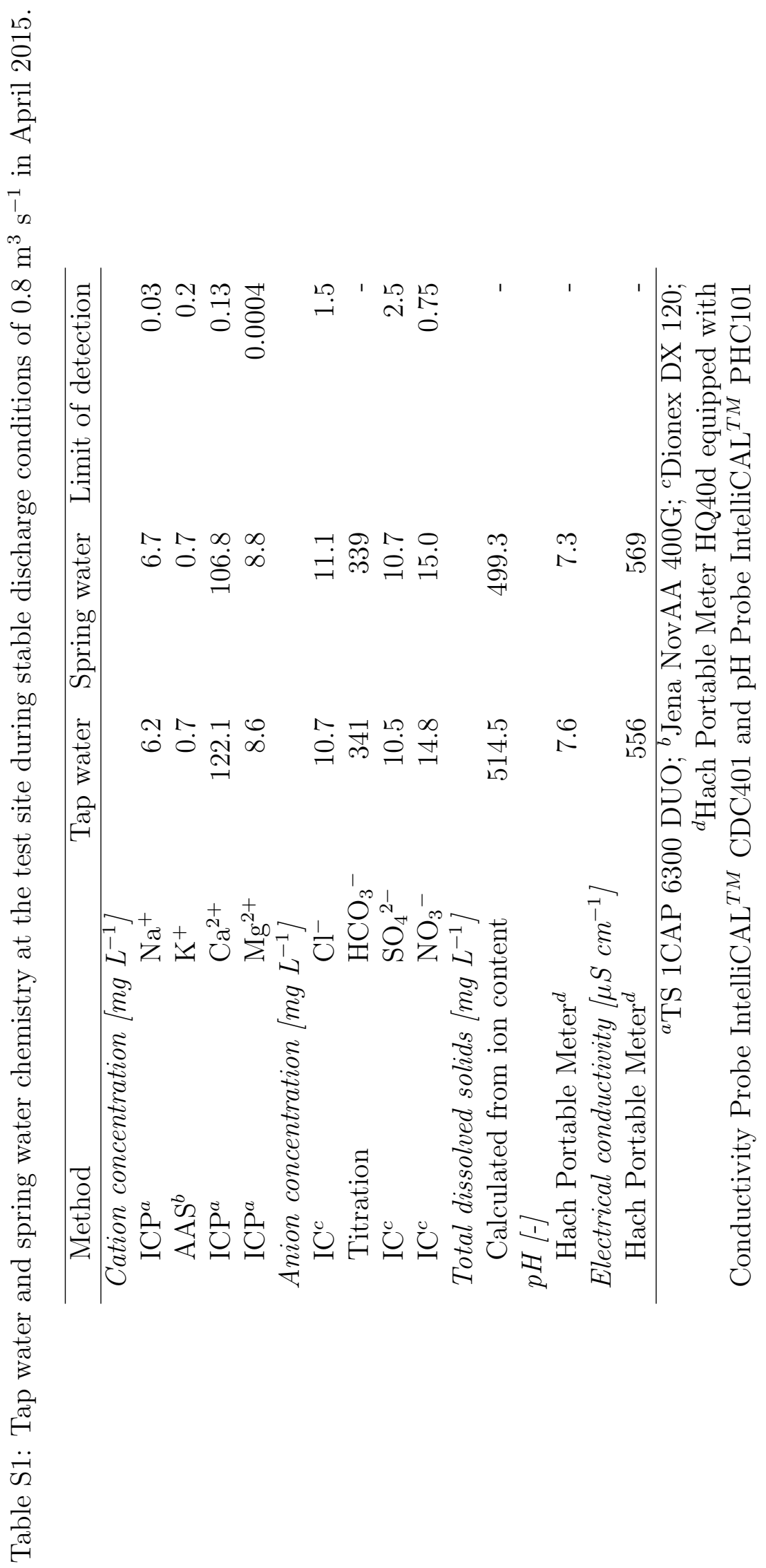



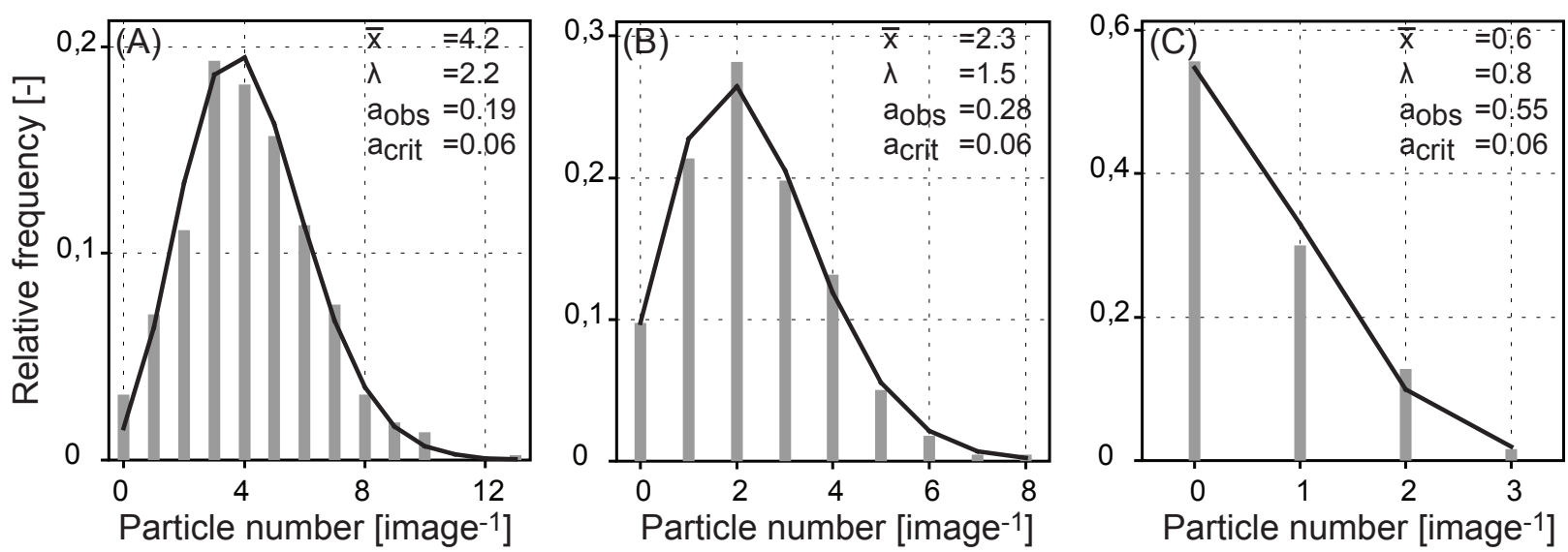

Figure S1: Probability density functions of particle distributions on filters for three examples with mean particle concentrations per image $(\bar{x})$ of $(\mathrm{A}) 4.2$, (B) 2.3, and (C) 0.6. Calculated values for a Kolmogorov-Smirnov test are well above the critical values at a significance level of $\alpha=5 \%$ and $n=400\left(\lambda=\right.$ standard deviation; $\mathrm{a}_{o b s}=$ Kolmogorov-Smirnov values; $\mathrm{a}_{\text {crit }}$ $=$ critical Kolmogorov-Smirnov values). 


\section{Silicate particles}
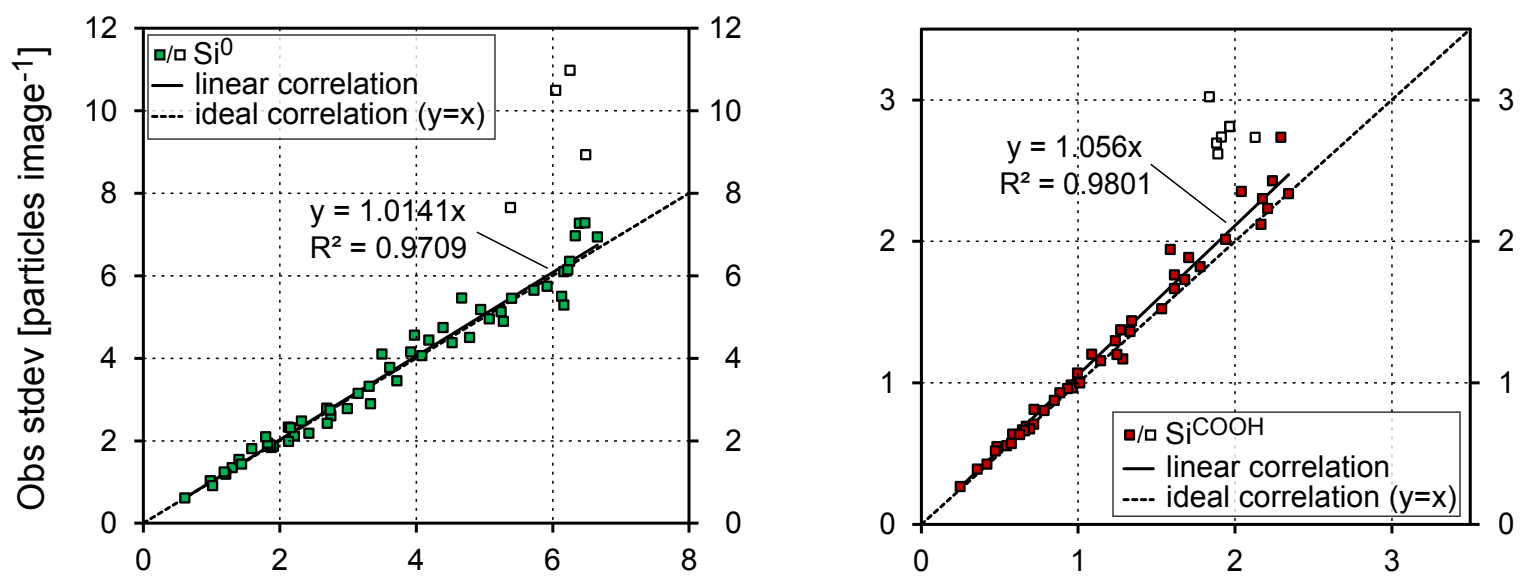

Polystyrene particles

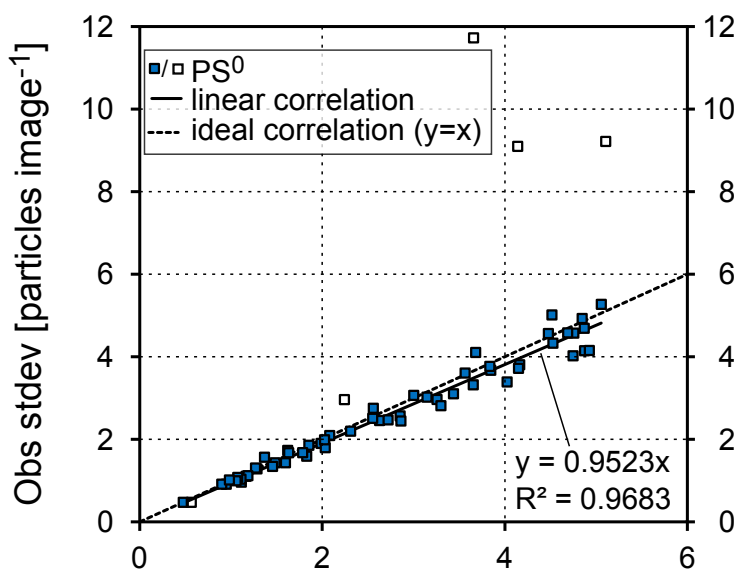

Nominal stdev [particles image ${ }^{-1}$ ]

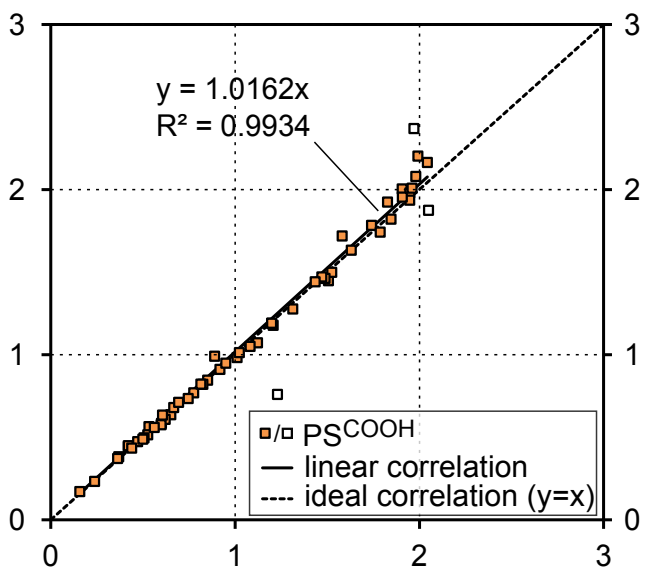

Nominal stdev [particles image ${ }^{-1}$ ]

Figure S2: Observed (obs) versus nominal standard deviations (stdev) of particle enumeration for each of the analyzed filters. Filled symbols show analysis that were considered for further processing, unfilled symbols show analysis that were excluded ( $\mathrm{Si}^{0}$ : unmodified silicate particles; $\mathrm{Si}^{\mathrm{COOH}}$ : carboxylated silicate particles; $\mathrm{PS}^{0}$ : unmodified polystyrene particles; $\mathrm{PS} C \mathrm{COH}$ : carboxylated polystyrene particles) 